ANNALES

POLONICI MATHEMATICI

XXIX (1975)

\title{
On two-point boundary value problems for systems of ordinary non-linear, first-order differential equations
}

\author{
by A. Lasota (Kraków)
}

\begin{abstract}
By using the Brouwer open mapping theorem, an existence theorem for the two-point boundary value problem is proved. The theorem is stated in the form of non-linear Fredholm alternative: the uniqueness of solutions implies the existence.
\end{abstract}

1. It is well known that for any system of ordinary linear differential equations the uniqueness of solutions of a linear boundary value problem implies the existence of solutions. It has also been discovered recently that the same is true for some boundary value problems related to nonlinear differential equations of the second [4] and the third order [2] (see also [1]). The purpose of this paper is to establish this kind of interdependence for a non-linear system

$$
x^{\prime}=f(t, x), \quad a \leqslant t \leqslant b,
$$

and the boundary value conditions

$$
A x(a)+B x(b)=r,
$$

where $r, x$ and $f$ are $d$-dimensional vectors and $A, B$ are $d \times d$-matrices. We shall assume that $f(t, x)$ is defined in the strip

$$
D=[a, b] \times R^{d}
$$

and satisfies the following condition:

(C) For every point $\left(t_{0}, r_{0}\right) \epsilon D$ there exists exactly one solution of equation (1), defined on $[a, b]$ and such that $x\left(t_{0}\right)=r$.

In the sequel $|\cdot|$ denotes the Euclidean norm in the space $R^{d}$. The norm in the space $R^{d \times d}$ of $d \times d$-matrices is defined by the formula:

$$
\|A\|=\sup \{|A x|:|x|=1\} .
$$

THEOREM 1. Let a triple $(f, \mathscr{A}, \mathscr{B})$ be given, where $f: D \rightarrow R^{d}$ is a continuous function satisfying condition (C) and where $\mathscr{A}, \mathscr{B}$ are sets of $d \times d$ matrices. Let at least one of the sets $A$ be open. 
Assume that for each $A \epsilon \mathscr{A}, B \in \mathscr{B}$ and $r \in R^{d}$ there exists at most one solution of the boundary value problem.(1)-(2). Then for each $A \epsilon \mathscr{A}, B \in \mathscr{B}$ and $r \in R^{d}$ there exists exactly one solution of (1)-(2).

Proof. Suppose that $\mathscr{A}$ is open. When $\mathscr{B}$ is open; the proof is similar. Fix $A \in \mathscr{A}, B \in \mathscr{B}$ and consider the mapping $u: R^{d} \rightarrow R^{d}$ given by the formula

$$
u(q)=A y(a, q)+B y(b, q),
$$

where $y(t, q)$ is the solution of equation (1) satisfying the initial condition $y(a, q)=q$. By assumption (C) such a solution exists and $u(q)$ is a continuous function of $q$. We claim that $u\left(R^{d}\right)=R^{d}$. Suppose that is not so and choose a point $p \& u\left(R^{d}\right)$. Let us write

$$
\begin{aligned}
\bar{t} & =\inf \left\{0 \leqslant t \leqslant 1:(1-t) u(0)+t p \notin u\left(R^{d}\right)\right\}, \\
\bar{p} & =(1-\bar{t}) u(0)+\bar{t} p .
\end{aligned}
$$

Since by the Brouwer open mapping theorem the set $u\left(R^{d}\right)$ is open, we have $\bar{t}>0$ and $\bar{p} q u\left(R^{d}\right)$. Now choose a sequence $t_{n} \rightarrow \bar{t}\left(0<t_{n}<\bar{t}\right)$ and write

$$
p_{n}=\left(1-t_{n}\right) u(0)+t_{n} p .
$$

From the definition of $\bar{t}$ it follows that $p_{n} \in u\left(R^{d}\right)$ and we may define the sequence $q_{n}=u^{-1}\left(p_{n}\right)$. We shall consider two cases:

$1^{\circ}$ The sequence $q_{n}$ is convergent to a point $\bar{q} \in R^{d}$.

$2^{\circ}$ The sequence $q_{n}$ is not convergent.

In the first case we have by the continuity of $u$

$$
u(\bar{q})=\lim _{n \rightarrow \infty} u\left(q_{n}\right)=\lim _{n \rightarrow \infty} p_{n}=\bar{p} .
$$

Thus $\bar{p}=u(q) \epsilon u\left(R^{d}\right)$, which is impossible. In the second case there exists a positive number $\varepsilon$ and two increasing sequences of integers $h_{n}$ and $k_{n}$ such that

$$
\left|q_{h_{n}}-q_{k_{n}}\right| \geqslant \varepsilon
$$

On the other hand, we have

$$
\left|u\left(q_{h_{n}}\right)-u\left(q_{k_{n}}\right)\right|=\left|p_{h_{n}}-p_{k_{n}}\right| \rightarrow 0 .
$$

Denote by $\delta$ the radius of a neighbourhood of the matrix $A$ which is contained in $\mathscr{A}$ and choose an integer $m$ such that

$$
\frac{\left|p_{h_{m}}-p_{k_{m}}\right|}{\left|q_{h_{m}}-q_{k_{m}}\right|}<\delta .
$$

It is easy to construct a matrix $\tilde{A}$ (not necessarily unique) which satisfies the following conditions:

$$
\tilde{A}\left(q_{h_{m}}-q_{k_{m}}\right)=p_{h_{m}}-p_{k_{m}}, \quad\|\tilde{\boldsymbol{A}}\|<\delta .
$$


From the definition of $p_{n}$ and $q_{n}$ it follows that

$$
A\left(q_{h_{m}}-q_{k_{m}}\right)+B\left(y\left(b, q_{h_{m}}\right)-y\left(b, q_{k_{m}}\right)\right)=p_{h_{m}}-p_{k_{m}} .
$$

Now write

$$
r_{m}=(A-\tilde{A}) y\left(a, q_{h_{m}}\right)+B y\left(b, q_{h_{m}}\right) .
$$

From (4), (5) and the identity $y(a, q)=q$ we obtain

$$
r_{m}=(A-\tilde{A}) y\left(a, q_{k_{m}}\right)+B y\left(b, q_{k_{m}}\right) .
$$

This means that $y\left(t, q_{k_{m}}\right)$ and $y\left(t, q_{h_{m}}\right)$ are two different solution of equation (1) satisfying the same boundary value condition of the form.

$$
(A-\bar{A}) x(a)+B x(b)=r_{m} .
$$

Since $A-\tilde{A} \epsilon \mathscr{A}$ and $B \in \mathscr{B}$, this contradicts the uniqueness. Thus the second case is also impossible. We have proved our claim that $u\left(R^{d}\right)=R^{d}$. Therefore for each $r \in R^{d}$ there exists a solution $q$ of the equation $u(q)=r$. The corresponding function $y(t, q)$ is the desired solution of the problem (1)-(2). The proof is complete.

2. It is easy to prove that the solution $x$ of the boundary value problem (1)-(2) in Theorem 1 depends continuously upon $A, B$ and $r$. We may state even a more general result. Denote by $\mathscr{F}$ the space of all continuous functions $f: D \rightarrow R^{d}$ with the topology of the uniform convergence on compact sets and denote by $C^{1}$ the space of all continuously differentiable functions $x:[a, b] \rightarrow R^{d}$ with the usual norm

$$
\|x\|_{C^{1}}=\sup \{|x(t)|: a \leqslant t \leqslant b\}+\sup \left\{\left|x^{\prime}(t)\right|: a \leqslant t \leqslant b\right\} .
$$

We have the following theorem:

THEOREM 2. Let a triple $\left.\mathscr{F}_{0}, \mathscr{A}, \mathscr{B}\right)$ be given, where $\mathscr{F}_{0}$ is a subset of $\mathscr{F}$ and where $\mathscr{A}, \mathscr{B}$ are sets of $d \times d$ matrices. Assume that

(i) for each $f \in \mathscr{F}_{0}$ condition (C) is satisfied,

(ii) at least one of the sets $\mathscr{A}, \mathscr{B}$ is open.

Assume, moreover, that for each $f \in \mathscr{F}_{0}, A \in \mathscr{A}, B \in \mathscr{B}$ and $r \in R^{d}$ there exists at most one solution of (1)-(2). Then for each $f \in \mathscr{F}_{0}, A \in \mathscr{A}, B \in \mathscr{P}$ and $r \in R^{d}$ there exists exactly one solution $x(t ; f, A, B, r)$ of $(1)-(2)$ and the mapping

$$
\mathscr{F}_{0} \times \mathscr{A} \times \mathscr{B} \times R^{d}{ }_{\ni}(f, A, B, r) \rightarrow x(\cdot ; f, A, B, r) \epsilon C^{1}
$$

is continuous.

Proof. The existence of the solution $x(\because ; f ; A, B, r)$ follows from. Theorem 1. In order to prove the continuity of the mapping (6): note that by the classical continuous dependence theorem the function $u=u(q ; f, A, B)$ defined by formula (3) is continuous. Since for each 
fixed triple $(f, A, B) \in F_{0}$ the mapping $q \rightarrow u(q, f, A, B)$ is a homeomorfism, it is easy to verify that the solution $q=q(f, A, B, r)$ of the equation $u=u(q ; f, A, B)=r$ depends continuously upon $(f, A, B, r)$. Thus the corresponding solution

$$
x(\cdot ; f, A, B, r)=y(\cdot ; q(f, A, B, r))
$$

of (1)-(2) is a continuous function of $(f, A, B, r)$.

3. Now we are going to apply Theorem 1 in two special cases. The first application is stimulated by an old paper of Krasnosielski-Pierov [3]; the second one is related to an example given in [5].

For $u, v \in R^{d}$ we denote by $\langle u, v\rangle$ the scalar product of $u$ and $v$. For any matrix $A \in R^{d}$ we write

$$
\begin{aligned}
& \|A\|_{0}=\|A\|=\sup \{|A x|:|x|=1\}, \\
& \|A\|_{1}=\inf \{|A x|:|x|=1\}, \\
& \|A\|_{2}=\sup \{\langle A x, x\rangle:|A x|=1\} \quad\left(\text { if }\|A\|_{0}>0\right) .
\end{aligned}
$$

THEOREM 3. Let $f: D \rightarrow R^{d}$ be a continuous function satisfying condition (C) and such that

$$
\langle f(t, x)-f(t, y), x-y\rangle \geqslant 0 \quad \text { for } t \in[a, b] ; x, y \in R^{d} .
$$

If $\|A\|_{0}<\|B\|_{1}$, then for each $r \in R^{d}$ the problem (1)-(2) has exactly one solution.

Proof. Fix $B$ and write

$$
\mathscr{A}=\left\{A \in R^{d \times d}:\|A\|_{0}<\|B\|_{1}\right\}, \quad \mathscr{B}=\{B\} .
$$

The set $\mathscr{A}$ is evidently open. Thus it remains to prove that for each $A \in \mathscr{A}$ and $r \in R^{d}$ the problem (1)-(2) admits at most one solution. Suppose, this is not so and let $x$ and $y$ be two different solutions of (1)-(2). From (1) it follows that

$$
\frac{d}{d t}|x-y|^{2}=2\left\langle x^{\prime}-y^{\prime}, x-y\right\rangle=2\langle f(t, x)-f(t, y), x-y\rangle \geqslant 0
$$

and consequently

$$
|x(a)-y(a)| \leqslant|x(b)-y(b)| .
$$

On the other hand, from (2) it follows that

and consequently

$$
A(x(a)-y(a))=B(y(b)-x(b))
$$

$$
\|A\|_{0}|x(a)-y(a)| \geqslant\|B\|_{1}|x(b)-y(b)| .
$$


From this and (7) we obtain

$$
\|\boldsymbol{A}\|_{0}|x(a)-y(a)| \geqslant\|B\|_{1}|x(a)-y(a)| .
$$

Since $x \not \equiv y$ and $f$ satisfies (C), we have $x(a) \neq x(b)$. Thus $\|A\|_{0} \geqslant\|B\|_{1}$, which contradicts the assumption.

THEOREM 4. Assume that $f: D \rightarrow R^{d}$ is continuous and satisfies the Lipschitz condition

$$
|f(t, x)-f(t, y)| \leqslant \varphi(t)|x-y| \quad \text { for } t \epsilon[a, b] ; x, y \in R^{d} .
$$

If

$$
\int_{a}^{b} \varphi(t) d t<\arccos \left\|-B^{-1} A\right\|_{2},
$$

then for each $r \in R^{d}$ the boundary value problem (1)-(2) admits exactly one solution.

Proof. Fix $B$ and write

$$
\mathscr{A}=\{A:(9) \text { holds }\}, \quad \mathscr{B}=\{B\} .
$$

The set $\mathscr{A}$ is open. Moreover, the continuity of $f$ and the Lipsohitz condition (8) imply (C). Thus in order to finish the proof it is sufficient to verify the uniqueness of (1)-(2) with $A \in \mathscr{A}$. Denote by $a$ and $y$ two different solutions of (1)-(2) and write $z=x-y$. From (1), (8) and (9). it follows that

$$
L \stackrel{\text { def }}{=} \int_{a}^{b}\left|\left(\frac{z}{|z|}\right)^{\prime}\right| d t \leqslant \int_{a}^{b} \frac{\left|z^{\prime}\right|}{|z|} d t \leqslant \int_{a}^{b} \varphi(t) d t<\arccos \left\|-B^{-1} A\right\|_{\mathbf{L}} .
$$

The integral $L$ denotes the length of the are

$$
\left\{\frac{z(t)}{|z(t)|}: a \leqslant t \leqslant b\right\}
$$

joining the vectors $z(a) /|z(a)|$ and $z(b) /|z(b)|$ on the unit sphere. The length of the shortest are which joins the same vectors is equal to

$$
\arccos \frac{\langle z(a), z(b)\rangle}{|z(a)||z(b)|} .
$$

Thus we have

$$
\arccos \frac{\langle z(a), z(b)\rangle}{|z(a)||z(b)|}<\arccos \left\|-B^{-1} A\right\|_{2}
$$

or

$$
\frac{\langle z(a), z(b)\rangle}{|z(a)||z(b)|}>\left\|-B^{-1} A\right\|_{2}
$$


On the other hand, from (2) it follows that $A z(a)=-B z(b)$ and consequently

$$
\frac{\left\langle-B^{-1} A z(a), z(a)\right\rangle}{\left|-B^{-1} A z(a)\right||z(a)|}=\frac{\langle z(a), z(b)\rangle}{|z(a)||z(b)|} .
$$

Now by the definition of $\|\cdot\|_{2}$ we obtain

$$
\frac{\langle z(a), z(b)\rangle}{|z(a)| z(b) \mid} \leqslant\left\|-B^{-1} A\right\|_{2},
$$

which contiradicts (10).

Added in proof: It was recently shown [6] that Theorem 1 and 2 may be stated for the system (1) and the boundary value condition $L x=r$, where $L$ is a linear operator from the space of continuously. differentiable functions defined on $[a, b]$ into $R^{d}$.

\section{References}

[1] P. Hartman, On n-parameter families and interpolation problems for nonlinear ordinary differential equations, Trans. Amer. Math. Soc. I54 (1971), p. 201-226.

[2] L. Jackson and K. Schrader, Existence and uniqueness of solutions of boun-: dary value problems for third order differential equations, J. Diff. Eq. 9 (1971), p. 46-54.

[3] М. А. К расносе льски и А. И. Перов, Об одном принчипе сущестөования ограниченных, периодических и почти-периодических решений у системы обыкновенных дифференчиальных уравнений, ДАН СССР 123 (1958), p. 235-238.

[4] A. L a sota and Z. Opial, On the existence and uniqueness of solutions of a boundary value problem for an ordinary second order differential equation, Colloq. Math. 18 (1967), p. $1-5$.

[5] - On the existence of solutions of linear problems for ordinary differential equations, Bull. Acad. Polon. Sci., Sér. sci. math., astronom. et phỳs. '14 (1966), p. $371-376$.

[6] A. Lasota and S. N. Chow, On boundary value problems for ordinary differential equations, J. Diff. Eq. 14 (1973), p. 326-337.

Regu par la Rédaction le 11.,10., 19.73. 